\title{
3 Research Square

\section{A scoring model to predict the prognosis of patients with poor-grade aneurysmal subarachnoid hemorrhage}

\section{Jie Shen}

Zhejiang University School of Medicine First Affiliated Hospital Jianbo Yu

Zhejiang University School of Medicine First Affiliated Hospital

Sicong Huang

Zhejiang University School of Medicine First Affiliated Hospital

\section{Rajneesh Mungur}

Zhejiang University School of Medicine First Affiliated Hospital

\section{Kaiyuan Huang}

Zhejiang University School of Medicine First Affiliated Hospital

\section{Xinfa Pan}

Zhejiang University School of Medicine First Affiliated Hospital

\section{Guofeng Yu}

People's Hospital of Quzhou

\section{Zhikai Xie}

Zhejiang University School of Medicine First Affiliated Hospital

\section{Lihui Zhou}

Zhejiang University School of Medicine First Affiliated Hospital

\section{Zongchi Liu}

Zhejiang University School of Medicine First Affiliated Hospital

\section{Dexin Cheng}

Zhejiang University School of Medicine First Affiliated Hospital Jianwei Pan

Zhejiang University School of Medicine First Affiliated Hospital

\section{Renya Zhan ( $\nabla 1196057 @ z j u . e d u . c n)$}

Zhejiang University School of Medicine First Affiliated Hospital

\section{Research article}

Keywords: Poor-grade aneurysmal subarachnoid hemorrhage (aSAH), scoring system, prognosis 
Posted Date: September 2nd, 2020

DOl: https://doi.org/10.21203/rs.3.rs-69333/v1

License: (c) (i) This work is licensed under a Creative Commons Attribution 4.0 International License. Read Full License 


\section{Abstract \\ Background}

Patients with poor-grade aneurysmal subarachnoid hemorrhage (aSAH), defined as World Federation of Neurosurgical Societies (WFNS) grade IV-V has high rates of disability and mortality. The objective of this research is to prognosticate outcomes of poor-grade aSAH accurately.

\section{Methods}

A total of 147 poor-grade aSAH patients in our center were enrolled. Risk variables identified by multivariate logistic regression were used to devise the scoring model (total score of 0-9 points). The score values were estimated according to $\beta$ coefficients. A cohort of 68 patients from another institute was used to validate the model.

\section{Results}

Multivariate analysis revealed that modified Fisher grade above II (odds ratio [OR], 2.972; $p=0.034$ ), age $\geq 65$ years $(O R, 3.534 ; p=0.006)$, conservative treatment $(O R, 5.078 ; p=0.019)$, WFNS Grade $V(O R$, 2.638; $p=0.029)$, delayed cerebral ischemia $(O R, 3.170 ; p=0.016)$, shunt-dependent hydrocephalus (OR, 3.202; $p=0.032)$ and cerebral herniation $(O R, 7.337 ; p<0.001)$ were significant predictors of poor prognosis (modified Rankin Scale $[\mathrm{mRS}] \geq 3$ ). By integration of above factors, a scoring system was constructed and divided poor-grade aSAH patients into three categories: low risk ( $0-1$ point), intermediate risk (2-3 points) and high risk (4-9 points), with risk of poor prognosis being $11 \%, 52 \%$ and $87 \%$ respectively $(P<0.001)$. The area under the curve in derivation cohort was $0.844(p<0.001 ; 95 \% \mathrm{Cl}$, $0.778-0.909)$. AUC in validation cohort was 0.831 ( $p<0.001 ; 95 \% \mathrm{Cl}, 0.732-0.929)$.

\section{Conclusions}

The new scoring model could improve prognostication of prognosis and help decision-making for subsequent complement treatment.

\section{Background}

Intracranial aneurysms are abnormal protrusion of the intracranial arterial wall caused by various reasons $[1,2]$. The prevalence rate of intracranial aneurysms in global population (average 50 years old) can reach $3.2 \%$ [3]. And literatures reported about 1 to $2 \%$ of aneurysms would ruptured[4]. According to statistics, the global incidence of aneurysmal subarachnoid hemorrhage (aSAH) is 9-11 per 100000 person years worldwide. And poor-grade aSAH (World Federation of Neurological Surgeons Grades IV and V) comprises $18-30 \%$ among all aSAH patients $[5,6]$. Patients with poor-grade aSAH are more critically ill, 
with moderate to severe hemiplegia, loss of consciousness, even deep coma, and with higher risk for developing intracranial hematoma, diffuse cerebral edema and cerebral vasospasm. The meta-analysis published by Han et al. reported a $26 \%$ mortality rate of poor-grade aSAH[7]. Existing literature also indicates that the disability rate of poor-grade aSAH exceeds $60 \%[8]$.

For poor-grade aSAH patients, a comprehensive prediction for prognosis is essential. The earlier International Subarachnoid Aneurysm Trial (ISAT) could achieve an accurate prediction of 60 days casefatality after aneurysmal subarachnoid hemorrhage[9]. And the Subarachnoid Hemorrhage International Trialists (SAHIT) model was highly predictive of outcome and used to counsel all patients with aSAH and their family members[10]. The external validation of SAHIT Predictive Model using Barrow Ruptured Aneurysm Trial (BRAT) Cohort and final result showed its AUC of unfavorable outcome was 0.734[11]. However, these studies included patients exposed to different subgroups of various treatment procedures, while most of them were good-grade aSAH patients who were eligible for surgical treatment. Good-grade aSAH and poor-grade aSAH differ in disease progression and survival prognosis[5, 12]. Previous studies usually combine these two groups for analysis without detailed stratifying[9-11]. Therefore, these predictive models have some limitations in accurately predicting the outcomes of poor-grade aSAH patients. The objective of our research was to devise a new scoring system to evaluate the prognosis of patients with poor-grade aSAH intuitively.

\section{Methods}

\section{Study Design}

The derivation cohort included poor-grade aSAH patients diagnosed and treated at the department of neurosurgery of our center from January 2013 to January 2019. Validation cohort was collected at the department of neurosurgery of another institute from January 2016 to January 2019. The inclusion criteria were as follows: (1) aSAH was diagnosed by computed tomography (CT) or lumbar puncture in medical center; (2) aneurysms were confirmed as the cause of SAH on digital subtraction angiography (DSA), three-dimensional CT angiography or magnetic resonance angiography (MRA); (3) WFNS grade of level 4 or level 5 ; (4) family members of patients signed informed consent to cooperate with clinical treatment procedures; (5) patients without surgical treatment in referral centers. The exclusion criteria were: (1) traumatic, mycotic, or arteriovenous malformation-related aneurysms or SAH of unknown etiology; (2) WFNS grade or Hunt-Hess grade lower than level 3; (3) absence of patients' important medical information; (4) patients treated by medical instruments or drugs which were not approved. The STROBE statement guideline has been implemented in this manuscript.

\section{Clinical Therapeutic Protocol}

Patients admitted in emergency conditions all accepted early resuscitation, early CT angiography, multidisciplinary consensus consultation, conservative treatment or surgical treatment. A multidisciplinary team of neurosurgeons and anesthesiologists made therapeutic decisions by taking clinical conditions and families' consent into account. The treatment mode in our study was divided into 
two categories: (1) Conservative group: patients received pure medicinal conservative treatment and could also be patients received other basic surgical methods but without treating responsible aneurysms, such as external drainage surgery, hematoma evacuation and decompressive craniectomy. (2) Clipping or Coiling group: patients undergoing primary aneurysm embolization or clipping only or combined with basic surgical operation on the basis of coiling or clipping. All patients who decided to undergo surgical treatment followed an early treatment strategy (within 72 hours). Antiplatelets were administered to prevent thrombosis after stent-assisted embolization and nimodipine were routinely used to prevent and treat cerebral vasospasm.

\section{Clinical Data and Variables Definition}

Patients' clinical variables were divided into six categories: (1) Demographic characters: gender and age. Age was divided into two subcategories by the demarcation line of 65 years old. (2) Medical history: hyperlipidemia, hypertension, diabetes mellitus, history of cerebrovascular disease, alcohol consumption and smoking. (3) Radiologic Imaging and laboratory examination: white blood cell count (WBC) $\geq$ $15 \times 10^{9}$, intracerebral hemorrhage (ICH), intraventricular hemorrhage (IVH), ventricular casting, WFNS grade and modified Fisher grade. Modified Fisher grade was divided into two subcategories by the demarcation line of grade II. (4) Aneurysm morphology: wide-necked aneurysms (aneurysms with a neck width $\geq 4 \mathrm{~mm}$ or a neck ratio greater than 1:2), multiple aneurysms, aneurysm size $(\mathrm{mm})$, location of Aneurysm. (5) Treatment: clipping, coiling, conservative group and continuous lumbar subarachnoid drainage. (6) Complication: acute hydrocephalus, shunt-dependent hydrocephalus, aneurysm rebleeding, epilepsy, pulmonary infection, intracranial infection, cerebral vasospasm (CVS), delayed cerebral ischemia (DCl) and cerebral herniation. CVS was defined as arterial stenosis found by CTA examination when the patient's clinical symptoms deteriorated; Or vasospasm detected during DSA[13]. DCI was defined as: 1. The occurrence of focal neurological impairment, or a decrease of at least 2 points on the Glasgow Coma Scale, and this situation cannot be attributed to other cause, such as cerebral rebleeding or encephaledema; 2. A new low-density area that had not been seen on previous CT scan and not attributable to other causes such as surgical treatment, or low-density shadow after absorption of hematoma[14].

\section{Outcomes Measure}

A dynamic follow-up evaluation was performed at 6 months after surgery by neurosurgeons according to modified Rankin score (mRS) via telephone or outpatient appointment. The assessment of patients' neurological prognosis was mainly focused on whether presented with the absence of self-care ability or not. Functional prognosis was classified as good (mRS scores $0-2$ ) or poor (mRS scores $3-6$ ).

\section{Statistical Analysis}

Data were analyzed with SPSS, Version 23.0 (IBM, Armonk, New York). Continuous variables were reported as a mean \pm standard deviation and compared between favorite and poor outcomes using unpaired $\mathrm{T}$ test. Categorical variables were reported as a proportion and percentile and were analyzed 
using $\mathrm{X}^{2}$ or Fisher exact test, as appropriate. Univariate and multivariate logistic regression analyses were performed using the poor outcomes as the outcome variable in the derivation cohort. Variables with $\mathrm{P}$ value $\leq 0.1$ in univariate analysis were entered into multivariate logistic regression with stepwise backward selection. Risk variables independently associated with prognosis were entering into the new scoring model. The points of each factors were assigned based on the corresponding $\beta$ coefficient in the multivariate analysis. The discrimination of prognostic models was assessed by area under the receiver operating characteristic curves (AUC). Hosmer-Lemeshow goodness of fit test and calibration plot were used to evaluate the calibration of prediction model.

\section{Results}

\section{Basic Information of Patients}

The detailed process of selection and exclusion of patients in modeling group and validation group was shown in Figure 1. In total, 147 patients were included in the derivation study, while 68 patients were included in the validation cohort.

In the derivation cohort, $55(37 \%)$ patients were male and $92(63 \%)$ were female. The age of cases ranged from 37 to 87 , the mean age was $61.3 \pm 11.5$ years, and approximately $39 \%$ of patients were over 65 years old. The baseline characteristics of the 147 patients with poor grade aSAH are presented in Table 1. A total of $124(84.3 \%)$ patients accepted surgical therapies including coiling $(29.2 \%)$ and clipping (55.1\%). The rest of $23(15.7 \%)$ patients accepted conservative treatments. The distribution of mRS score of 147 poor-grade aSAH patients accepted different treatment was shown in Figure 2 (A). In Figure 2 (B, $C, D, E)$, patients accepted coiling or clipping had a better prognosis than patients accepted conservation treatment, but there was no statistical significance of prognosis between patients accepted coiling and clipping. There were 114 (77.6\%) poor-grade aSAH patients with modified fisher grade above 2 and 85 $(57.8 \%)$ patients were recorded with WFNS grade V. And the distribution of mRS score of 147 poor-grade aSAH patients with different modified Fisher grade was shown in Figure $2(F)$. Figure $2(G, H, I, J)$ reflects the influence of different modified Fisher grade groups on the prognosis of patients. During the 6 months' follow-up after discharge, 85 patients (58\%) had poor outcomes.

Of the 68 patients in the validation cohort, $25(36.7 \%)$ patients were over 65 years old. A total of 44 $(64.7 \%)$ poor-grade aSAH patients with a modified fisher grade above 2 and $40(58.8 \%)$ patients presented with WFNS grade V. Forty-nine (72\%) patients accepted surgical therapies. During the 6 months' follow-up after discharge, 38 patients (56\%) had poor outcomes. Specific data of validation cohort is presented in Table 1.

\section{Univariate Analysis of Poor Outcome}

The association between clinical variables and poor outcomes identified by univariate analysis are shown in Table 1. The poorer prognosis is associated with elderly age (age $\geq 65)(P=0.027)$, intraventricular hemorrhage $(P=0.005)$, WFNS grade $V(P<0.001)$, conservative treatment $(P=0.009)$, 
modified fisher grade $>2(P=0.001)$, emergence of cerebral herniation $(P<0.001)$, aneurysm rebleeding $(P=0.004)$, CVS $(P=0.041)$ and $D C l(P=0.030)$. Medical history of patients and data of aneurysm were not significantly interrelated with clinical outcome (Table 1$)$.

\section{Multivariate Regression Analysis of Poor Outcome}

Ten variables $(P<0.1$ in univariate analysis) were entered into multivariate logistic regression. Table 2 showed age $\geq 65$ years $(O R, 3.534 ; p=0.006)$, modified Fisher grade $>2(O R, 2,972 ; p=0.034)$, cerebral herniation (OR, 7.337; $p<0.001)$, WFNS V $(O R, 2.638 ; p=0.029), S D H(O R, 3.202 ; p=0.032)$, conservative treatment $(\mathrm{OR}, 5.078 ; \mathrm{p}=0.019)$ and $\mathrm{DCl}(\mathrm{OR}, 3.170 ; p=0.016)$ are independent risk factors for poor outcomes. The Hosmer-Lemeshow test reflects a satisfied degree of consistency between the predicted risk of the model and the actual risk $(P=0.589$, Table 2$)$.

\section{Development of the Scoring System}

By integration of 7 independent risk factors including modified Fisher grade above grade II, age $\geq 65$ years, conservative treatment, WFNS grade $\mathrm{V}, \mathrm{DCl}, \mathrm{SDH}$, and cerebral herniation, a scoring system referred to as Poor-grade Aneurysmal Subarachnoid Hemorrhage Prognostic Scoring System (PASHPSS below) was then constructed (Table 3). Based on the $\beta$ coefficient in the multivariate analysis, a score of 2 was assigned to cerebral herniation and conservative treatment respectively; a score of 1 was assigned to each other risk factors respectively; otherwise, 0 point was assigned. According to the sum of the scores (range from 0 - 9), the new model divided poor-grade aSAH patients into three prognostically different categories (Table 4): the predict risk of poor prognosis is $11 \%$ in patients with a total point of 0 - 1 (low risk category), $51 \%$ in those with a total point of 2 - 3 (intermediate risk category) and $87 \%$ in those with a total point of four or above (high risk category).

\section{Discrimination and Calibration of the Scoring System}

In modeling cohort, the AUC of PASHPSS was 0.844 (95\% Cl: $0.778-0.909$; Figure 3$)$. And the HosmerLemeshow test showed a good calibration $(P=0.589)$.

The PASHPSS also showed good discrimination and good calibration in the validation cohort, with an AUC of $0.831(95 \% \mathrm{Cl}, 0.732-0.929$, Figure 3$)$ and Hosmer-Lemeshow test fit well $(P=0.984)$. And in validation cohort (Table 3), the observed risks in the three risk groups were also closed to the predicted risks: low risk (actual observed risk of poor prognosis, 19\%), intermediate risk (actual observed risk of poor prognosis, $48 \%$ ) and high risk (actual observed risk of poor prognosis, $81 \%$ ).

\section{Discussion}

As serious cerebrovascular disease, poor-grade aneurysmal subarachnoid hemorrhage has high rates of mortality and disability. In this study, the poor prognosis of patients in the modeling cohort and validation cohort were both more than $55 \%$. Though active and effective treatments is provided, some aSAH patients still present neurological dysfunction and life disorders, which would bring heavy impact on 
society and families $[1,4,6,8]$. It is necessary to explore relevant risk factors and evaluate the prognosis of these patients. Several modifiable and non-modifiable risk factors for poor prognosis of poor-grade aSAH patients were known today, the most common risk factors were older age, cerebral herniation, WFNS grade $V$, higher modified Fisher grade[9, 10, 12, 15-19]. These risk factors were also identified in our study.

The choice of treatment methods is significantly related to the prognosis of patients with poor-grade aSAH. In a systematic review of 815 patients with aSAH, researchers reported that the good prognosis of clipping, embolization and conservative treatment were $45.3 \%, 36.3 \%$ and $9.0 \%$, respectively[20]. In our study, the adverse prognosis of patients treated with clipping or coiling were significantly lower than those treated with conservative therapy and there was no statistically significant difference in prognosis between clipping and embolization. Combining our center's experience with previous literature, as far as poor-grade aSAH patients are concerned, more aggressive treatment of responsible aneurysms through surgery has better therapeutic prognosis compared to conservative treatment.

Postoperative complications also played an important role in affecting the prognosis of poor-grade aSAH patients. As a critical complication, aneurysmal rebleeding usually causes the sharp rise of intracranial pressure, damages patient's nerve function and increases the risk of death in short term[21-23]. Cerebral vasospasm is generally considered as a risk factor for poor prognosis. But immediate vasospasm is usually difficult to detect. And nimodipine has been routinely used in clinical to prevent occurrence of cerebral vasospasm, leading to a further reduction in the incidence of cerebral vasospasm[24]. More commonly observed and easier detected complication during clinical treatment is delayed cerebral ischemia caused by cerebral vasospasm, which is a strong independent risk factor of poor prognosis of patients with poor-grade aSAH[14, 25,26]. Delayed cerebral ischemia continues to be an important cause of cognitive impairment and disability after aSAH despite accepted aggressive management[27-29]. A single center study including 888 aSAH patients found that shunt dependent hydrocephalus was a strong independent risk factor for unfavorable functional outcome[30]. Our final results also confirm the predictive role of above-mentioned factors.

There are also some other risk factors that have been raised in recent articles but have not been widely recognized. Intraventricular hemorrhage was also regarded as a risk factor for poor outcomes in many literatures[31]. It has been verified in our univariate analysis, however, eliminated in final multivariate regression analysis. The possible reason might be that intraventricular hemorrhage caused impairment of cerebrospinal fluid absorption by blocking arachnoid villi and brain capillaries, which affected the prognosis by developing into chronic hydrocephalus[32,33]. Whether or not the location and size of the aneurysm are predictive factors for the poor prognosis of aSAH is still inconclusive[19]. These inconsistent results may be explained by the treatment selection bias in different studies. And there is no connection between the location and size of the aneurysm and long-term prognosis according to our data. In a multicenter study about poor-grade aSAH, Zhao et al. pointed out that wide necked aneurysms and postoperative pneumonia were also poor prognostic factor[16]. But these two risk factors haven't 
been identified in our study. Leucocytosis (WBC > 15 $\times 109 / \mathrm{L})$ was regarded as a predictive factor for of poor prognosis in a 9-year cohort study[17], however, it had not been reported in other articles.

The current literature about poor-grade aSAH focused on reporting risk factors of prognosis in this group of patients, however, prognostic predictive models for this group of patients are rare. A recent systematic review assessed 11 clinical prediction models for aneurysmal subarachnoid hemorrhage and pointed out that the most common factors associated with outcome were age (8 of 11 studies), admission neurologic grade on admission ( 10 of 11 studies), and amount of blood detected by CT scan upon admission ( 6 of 11 studies)[19]. Although WFNS and modied Fisher grade scales are commonly used, both scales are not absolutely reliable in patients because of the subjective nature of the parameters on which they were built[34]. For example, WFNS or Hunt-Hess scales are generally unreliable in intubated patients. In addition, in two articles that established predictive scores in poor-grade aSAH patient populations, these factors were also applied; but no additional risk factors were added to circumvent the errors caused by inter- and intrarater variability in part $[17,18]$. Undeniably, more valuable risk variables added into the risk score could improve its predictivity. Treatment methods, shunt-dependent hydrocephalus and delayed cerebral ischemia are three factors that affect the long-term neurological prognosis and cognitive impairment of patients, their role in predicting the prognosis of patients is worthy of recognition[14, 30 , $32,35]$. Our PASHPSS have significantly improved its discrimination comparing to other risk scores by drawing into these risk factors. For example, the AUC of SAHIT model was 0.734 , while the AUC of WAP score which established on poor-grade aSAH patients was 0.74. But the AUC of PASHPSS was 0.844, which can be regarded as excellent, especially when predicting the prognosis of poor-grade aSAH.

At present, several studies have proposed the prognosis model of poor-grade aSAH, but most of them have limitations in reporting calibration, discrimination and external validation. Clinicians generally do not use existing models for predicting the prognosis of poor-grade aSAH, even the internal effectiveness of them are not inferior to PASHPSS[17-19], partly because they lack of external validity. However, PASHPSS showed good discrimination in the validation data; its area under the curve was 0.831 , in other words, still performs well when it was applied to a new patient cohort different from the original cohort.

This study shows that the PASHPSS devised by the risk factors predicts the future risk of poor prognosis well. And it could help guide clinical decisions and patient consultation and also reduce the cost of treatment by ensuring effective resource allocation. Such benefits may be particularly important in the management of patients with poor-grade aneurysmal subarachnoid hemorrhage.

\section{Limitations}

Some limitations of our risk score need to be discussed. First, statistical data were retrospectively collected. Second, the results of this study only represent the subgroup of poor-grade aSAH patients. Therefore, the scoring model is applicable to the prediction of poor prognosis risk only among poor-grade aSAH patients. With regard to functional neurologic outcomes, we selected six months after discharge as the follow-up cut-off points which is the critical period of neurological recovery, however, if, data on long- 
term follow-up could be acquired, the prediction of prognosis will be more accurate. Furthermore, the modeling data was acquired from a single center, these may lead to some inevitable bias of analysis and conclusions.

\section{Conclusion}

The obtained results have allowed us to draw the following conclusions. The main risk factors affecting the prognosis of patients with poor-grade aSAH are modified Fisher grade, age, therapeutic schedule, WFNS grade, delayed cerebral ischemia, shunt-dependent hydrocephalus and cerebral herniation. The PASHPSS is an efficient tool for predicting the prognosis of poor grade aneurysm, easily measured and helpful to make the decision of subsequent complement treatment and reduce the cost of treat by ensuring effective resource allocation.

\section{Abbreviations}

Aneurysmal Subarachnoid Hemorrhage (aSAH). World Federation of Neurosurgical Societies (WFNS). Odds Ratio (OR). Confidence Interval (CI). Intensive Care Unit (ICU). Delayed Cerebral Ischemia (DCI). Cerebral vasospasm (CVS). Glasgow Outcome Scale (GOS). Intraventricular Hemorrhage (IVH). Intracerebral Hemorrhage (ICH). Computed Tomography (CT). Modified Rankin score (mRS). International Subarachnoid Aneurysm Trail (ISAT). Subarachnoid Hemorrhage International Trialists (SAHIT). Barrow Ruptured Aneurysm Trial (BRAT). Continuous Lumber Subarachnoid Drainage (CLSD). Area under the curve (AUC). White blood cell count (WBC). Shunt-dependent hydrocephalus (SDH). Poor-grade Aneurysmal Subarachnoid Hemorrhage Prognostic Scoring System (PASHPSS).

\section{Declarations}

All authors read and approved the final manuscript. This manuscript has not been published elsewhere and is not under consideration

\section{Consent for publication}

Not applicable

\section{Ethics approval and consent to participate}

Ethics approval has been obtained from the ethics committee of First Affiliated Hospital of Zhejiang University.

\section{Availability of data and material}

All data of this research is included in this published article.

\section{Competing interests}


All authors declare that they have no competing interests.

\section{Reporting checklist}

STROBE checklist

\section{Funding}

This research was funded by "Key Research \& Development (R\&D) Plan of Zhejiang Province (NO. 2019c03034)".

\section{Authors' contributions}

JS and J-B Y contributed to manuscript writing, acquisition of the data, analysis and interpretation of the data.

S-C H contributed to acquisition of follow-up data and preliminary revision of manuscript content.Rajneesh Mungur as a colleague whose native language is English, contributed to correct the English-language usage in manuscripts.

$\mathrm{K}-\mathrm{Y} \mathrm{H}$ contributed to acquisition of the data, preliminary revision of manuscript content.

X-F P contributed to preliminary revision of manuscript content.

G-F Y contributed to provide the external validation data.

Z-K X, L-H Z, Z-C L and D-X C contributed to review the literature.

J-W P and R-Y Z contributed to critical revision of the manuscript for intellectual content.

\section{Acknowledgements}

Thanks for the data provided by An Wu, director of the Department of Neurosurgery of The Quzhou Municipal People's Hospital.

\section{References}

1. de Rooij NK, Linn FH, van der Plas JA, Algra, ARinkel, GJ. Incidence of subarachnoid haemorrhage: a systematic review with emphasis on region, age, gender and time trends. J Neurol Neurosurg Psychiatry. 2007;78(12):1365-72.

2. Wiebers DO. Unruptured intracranial aneurysms: natural history, clinical outcome, and risks of surgical and endovascular treatment. The Lancet. 2003;362(9378):103-10.

3. Thompson BG, Brown RD Jr, Amin-Hanjani S, et al. Guidelines for the Management of Patients With Unruptured Intracranial Aneurysms: A Guideline for Healthcare Professionals From the American 
Heart Association/American Stroke Association. Stroke. 2015;46(8):2368-400.

4. Ellis JA, Nossek E, Kronenburg A, Langer, DJOrtiz, RA. Intracranial Aneurysm: Diagnostic Monitoring, Current Interventional Practices, and Advances. Curr Treat Options Cardiovasc Med. 2018;20(12):94.

5. Steiner T, Juvela S, Unterberg A, et al. European Stroke Organization guidelines for the management of intracranial aneurysms and subarachnoid haemorrhage. Cerebrovasc Dis. 2013;35(2):93-112.

6. Howard., BMBarrow., DL. Outcomes for Patients with Poor-Grade Subarachnoid Hemorrhage: To Treat or Not To Treat? World Neurosurgery. 2016;85:125-9.

7. Han Y, Ye F, Long X, et al. Ultra-Early Treatment for Poor-Grade Aneurysmal Subarachnoid Hemorrhage: A Systematic Review and Meta-Analysis. World Neurosurg. 2018;115:e160-e71.

8. Wartenberg KE. Critical care of poor-grade subarachnoid hemorrhage. Curr Opin Crit Care. 2011;17(2):85-93.

9. Risselada R, Lingsma HF, Bauer-Mehren A, et al. Prediction of 60 day case-fatality after aneurysmal subarachnoid haemorrhage: results from the International Subarachnoid Aneurysm Trial (ISAT). Eur J Epidemiol. 2010;25(4):261-6.

10. Jaja BNR, Saposnik G, Lingsma HF, et al. Development and validation of outcome prediction models for aneurysmal subarachnoid haemorrhage: the SAHIT multinational cohort study. BMJ. 2018;360:j5745.

11. Mascitelli JR, Cole T, Yoon S, et al. External Validation of the Subarachnoid Hemorrhage International Trialists (SAHIT) Predictive Model Using the Barrow Ruptured Aneurysm Trial (BRAT). Cohort Neurosurgery. 2020;86(1):101-6.

12. Molyneux A. International Subarachnoid Aneurysm Trial (ISAT) of neurosurgical clipping versus endovascular coiling in 2143 patients with ruptured intracranial aneurysms: a randomised trial. The Lancet. 2002;360(9342):1267-74.

13. Frontera JA, Fernandez A, Schmidt JM, et al. Defining vasospasm after subarachnoid hemorrhage: what is the most clinically relevant definition? Stroke 2009;40(6):1963-8.

14. Vergouwen MD, Vermeulen M, van Gijn J, et al. Definition of delayed cerebral ischemia after aneurysmal subarachnoid hemorrhage as an outcome event in clinical trials and observational studies: proposal of a multidisciplinary research group. Stroke. 2010;41(10):2391-5.

15. Molyneux AJ, Birks J, Clarke A, Sneade, MKerr, RSC. The durability of endovascular coiling versus neurosurgical clipping of ruptured cerebral aneurysms: 18 year follow-up of the UK cohort of the International Subarachnoid Aneurysm Trial (ISAT). The Lancet. 2015;385(9969):691-7.

16. Zhao B, Yang H, Zheng K, et al. Preoperative and postoperative predictors of long-term outcome after endovascular treatment of poor-grade aneurysmal subarachnoid hemorrhage. J Neurosurg. 2017;126(6):1764-71.

17. Szklener S, Melges A, Korchut A, et al. Predictive model for patients with poor-grade subarachnoid haemorrhage in 30-day observation: a 9-year cohort study. BMJ Open. 2015;5(6):e007795. 
18. Zheng K, Zhong M, Zhao B, et al. Poor-Grade Aneurysmal Subarachnoid Hemorrhage: Risk Factors Affecting Clinical Outcomes in Intracranial Aneurysm Patients in a Multi-Center Study. Front Neurol. 2019;10:123.

19. Jaja BN, Cusimano MD, Etminan N, et al. Clinical prediction models for aneurysmal subarachnoid hemorrhage: a systematic review. Neurocrit Care. 2013;18(1):143-53.

20. Ohkuma H, Shimamura N, Naraoka, MKatagai, T. Aneurysmal Subarachnoid Hemorrhage in the Elderly over Age 75: A Systematic Review. Neurol Med Chir (Tokyo). 2017;57(11):575-83.

21. van Donkelaar CE, Bakker NA, Veeger NJ, et al. Predictive Factors for Rebleeding After Aneurysmal Subarachnoid Hemorrhage: Rebleeding Aneurysmal Subarachnoid Hemorrhage Study. Stroke. 2015;46(8):2100-6.

22. Larsen, CCAstrup, J. Rebleeding after aneurysmal subarachnoid hemorrhage: a literature review. World Neurosurg. 2013;79(2):307-12.

23. Lu VM, Graffeo CS, Perry A, et al. Rebleeding drives poor outcome in aneurysmal subarachnoid hemorrhage independent of delayed cerebral ischemia: a propensity-score matched cohort study. J Neurosurg 2019:1-9.

24. Findlay JM, Nisar, JDarsaut, T. Cerebral Vasospasm: A Review. Can J Neurol Sci. 2016;43(1):15-32.

25. Al-Mufti F, Roh D, Lahiri S, et al. Ultra-early angiographic vasospasm associated with delayed cerebral ischemia and infarction following aneurysmal subarachnoid hemorrhage. J Neurosurg. 2017;126(5):1545-51.

26. Leclerc JL, Blackburn S, Neal D, et al. Haptoglobin phenotype predicts the development of focal and global cerebral vasospasm and may influence outcomes after aneurysmal subarachnoid hemorrhage. Proc Natl Acad Sci U S A. 2015;112(4):1155-60.

27. Wang $X$, Han $C$, Xing D, Wang, CDing, $X$. Early management of poor-grade aneurysmal subarachnoid hemorrhage: A prognostic analysis of 104 patients. Clin Neurol Neurosurg. 2019;179:4-8.

28. Etminan N, Vergouwen MDRL. M. Angiographic vasospasm versus cerebral infarction as outcome measures after aneurysmal subarachnoid hemorrhage. Acta Neurochir. 2013;115:33-40.

29. Kreiter KT, Copeland D, Bernardini GL, et al. Predictors of cognitive dysfunction after subarachnoid hemorrhage. Stroke. 2002;33(1):200-8.

30. Paisan GM, Ding D, Starke RM, Crowley, RWLiu, KC. Shunt-Dependent Hydrocephalus After Aneurysmal Subarachnoid Hemorrhage: Predictors and Long-Term Functional Outcomes. Neurosurgery. 2018;83(3):393-402.

31. Nguyen HS, Li L, Patel M, Kurpad, SMueller, W. Radiodensity of intraventricular hemorrhage associated with aneurysmal subarachnoid hemorrhage may be a negative predictor of outcome. $J$ Neurosurg. 2018;128(4):1032-6.

32. de Oliveira JG, Beck J, Setzer M, et al. Risk of shunt-dependent hydrocephalus after occlusion of ruptured intracranial aneurysms by surgical clipping or endovascular coiling: a single-institution series and meta-analysis. Neurosurgery. 2007;61(5):924-33. 
33. Lai L.Morgan MK. Predictors of in-hospital shunt-dependent hydrocephalus following rupture of cerebral aneurysms. J Clin Neurosci. 2013;20(8):1134-8.

34. Degen LA, Dorhout Mees SM, Algra, ARinkel, GJ. Interobserver variability of grading scales for aneurysmal subarachnoid hemorrhage. Stroke. 2011;42(6):1546-9.

35. van Donkelaar CE, Dijkland SA, van den Bergh WM, et al. Early Circulating Lactate and Glucose Levels After Aneurysmal Subarachnoid Hemorrhage Correlate With Poor Outcome and Delayed Cerebral Ischemia: A Two-Center Cohort Study. Crit Care Med. 2016;44(5):966-72.

\section{Tables}


Table 1: Demographic and baseline characteristics of the study population and univariate analysis results of modeling cohorts

Variable
Modeling cohort

Favorite

Outcome

$\% /$

Mean \pm SD

No.of patients

$62(42 \%)$
Poor Outcome

$\%$ /

Mean \pm SD

85(58\%)

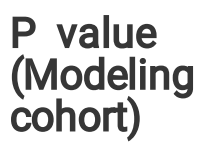

0.027

Age(years)

$\$ 65$

$\geqq 65$

Gender

Male

Female

Medical History

Hyperlipidemia

Hypertension

Diabetes

mellitus

Cerebrovascular $8(13 \%)$

disease

Alcohol

consumption

Smoking

15(24\%)

$45(53 \%)$

$40(47 \%)$

0.147

$36(42 \%)$

$49(58 \%)$

$26(31 \%)$

0.994

0.148

0.170

28(33\%)

$11(13 \%)$

0.995

$28(33 \%)$

$21(25 \%)$

0.943

0.110
$8(27 \%)$

10(33\%)

10(33\%)

$20(67 \%)$

$7(23 \%)$

$18(60 \%)$

$8(27 \%)$

$5(17 \%)$

Radiologic Imaging and laboratory examination

WBC $\geqq 15 \times 10^{9} \quad 30(48 \%)$

$\mathrm{ICH}$

IVH

Ventricular

Casting

modified Fisher

Grade
$21(34 \%)$

$41(66 \%)$

$42(49 \%) \quad 0.902$

0.448

0.005

0.195

$21(25 \%)$

10(16\%)

$34(40 \%)$

73(86\%)

0.001
$8(21 \%)$

21(55\%)

17(45\%)

16(42\%)

22(58\%)

11(29\%)

22(58\%)

$14(37 \%)$

$10(26 \%)$

$9(24 \%)$

19(50\%)

18(47\%)

$28(74 \%)$

$8(21 \%)$ 


\begin{tabular}{|c|c|c|c|c|c|}
\hline ॥ & $23(37 \%)$ & $12(14 \%)$ & & $15(50 \%)$ & $9(24 \%)$ \\
\hline III & $13(21 \%)$ & $32(38 \%)$ & & $5(17 \%)$ & $11(29 \%)$ \\
\hline IV & $26(42 \%)$ & $41(48 \%)$ & & 10(33\%) & $18(47 \%)$ \\
\hline WFNS & & & $<0.001$ & & \\
\hline IV & $38(61 \%)$ & $24(28 \%)$ & & $15(50 \%)$ & $13(34 \%)$ \\
\hline V & $24(39 \%)$ & $61(72 \%)$ & & $15(50 \%)$ & $25(66 \%)$ \\
\hline \multicolumn{6}{|c|}{ Aneurysm morphology } \\
\hline $\begin{array}{l}\text { Wide-necked } \\
\text { aneurysm }\end{array}$ & $31(50 \%)$ & $49(58 \%)$ & 0.317 & $13(43 \%)$ & $19(50 \%)$ \\
\hline $\begin{array}{l}\text { Multiple } \\
\text { aneurysms }\end{array}$ & $8(13 \%)$ & $16(19 \%)$ & 0.338 & $5(17 \%)$ & $10(26 \%)$ \\
\hline $\begin{array}{l}\text { Aneurysm size } \\
(\mathrm{mm})\end{array}$ & $5.9 \pm 3.3$ & $6.1 \pm 3.7$ & 0.860 & $6.5 \pm 2.5$ & $6.7 \pm 3.1$ \\
\hline $\begin{array}{l}\text { Location of } \\
\text { Aneurysm }\end{array}$ & & & 0.435 & & \\
\hline ICA & $13(21 \%)$ & $10(12 \%)$ & & $6(20 \%)$ & $5(13 \%)$ \\
\hline$A C$ & $6(10 \%)$ & $10(12 \%)$ & & $4(13 \%)$ & $3(8 \%)$ \\
\hline ACA & $12(19 \%)$ & $21(25 \%)$ & & $8(27 \%)$ & $8(21 \%)$ \\
\hline MCA & $15(24 \%)$ & $15(17 \%)$ & & $5(17 \%)$ & $9(24 \%)$ \\
\hline PCOM & $11(18 \%)$ & $16(19 \%)$ & & $5(17 \%)$ & $8(21 \%)$ \\
\hline PC & $5(8 \%)$ & $13(15 \%)$ & & $2(6 \%)$ & $5(13 \%)$ \\
\hline \multicolumn{6}{|l|}{ Treatment } \\
\hline $\begin{array}{l}\text { Therapeutic } \\
\text { Strategy }\end{array}$ & & & 0.009 & & \\
\hline Coiling & 19(31\%) & $24(28 \%)$ & & $16(53 \%)$ & $11(29 \%)$ \\
\hline Clipping & $39(63 \%)$ & $42(49 \%)$ & & $9(30 \%)$ & $13(34 \%)$ \\
\hline $\begin{array}{l}\text { Conservative } \\
\text { Treatment }\end{array}$ & $4(6 \%)$ & $19(23 \%)$ & & $5(17 \%)$ & $14(37 \%)$ \\
\hline CLSD & $21(34 \%)$ & 28(33\%) & 0.906 & $7(23 \%)$ & $9(24 \%)$ \\
\hline \multicolumn{6}{|l|}{ Complication } \\
\hline $\begin{array}{l}\text { Acute } \\
\text { hydrocephalus }\end{array}$ & $12(19 \%)$ & $20(24 \%)$ & 0.545 & $7(23 \%)$ & 10(26\%区 \\
\hline SDH & $8(13 \%)$ & $22(26 \%)$ & 0.054 & $7(23 \%)$ & $12(32 \%)$ \\
\hline
\end{tabular}




\begin{tabular}{|llllll|}
$\begin{array}{l}\text { Aneurysm } \\
\text { Rebleeding }\end{array}$ & $1(2 \%)$ & $13(15 \%)$ & 0.004 & 0 & $8(21 \%)$ \\
\hline Epilepsy & $4(7 \%)$ & $9(11 \%)$ & 0.383 & $1(3.3 \%)$ & $3(8 \%)$ \\
\hline $\begin{array}{l}\text { Pulmonary } \\
\text { Infection }\end{array}$ & $32(52 \%)$ & $50(59 \%)$ & 0.385 & $16(53 \%)$ & $21(55 \%)$ \\
$\begin{array}{l}\text { Intracranial } \\
\text { Infection }\end{array}$ & $33(53 \%)$ & $40(47 \%)$ & 0.460 & $13(43 \%)$ & $16(42 \%)$ \\
\hline CVS & $7(11 \%)$ & $21(25 \%)$ & 0.041 & $7(23 \%)$ & $16(42 \%)$ \\
\hline DCl & $13(21 \%)$ & $32(38 \%)$ & 0.030 & $6(20 \%)$ & $11(29 \%)$ \\
\hline $\begin{array}{l}\text { Cerebral } \\
\text { Herniation }\end{array}$ & $6(10 \%)$ & $38(45 \%)$ & $<0.001$ & $2(7 \%)$ & $16(42 \%)$ \\
\hline $\begin{array}{l}\text { ICH - Intracerebral Hemorrhage, IVH - Intraventricular Hemorrhage, WFNS - World Federation of } \\
\text { Neurosurgical Societies, CLSD - Continuous lumbar Subarachnod Drainage, SDH - Shunt-dependent } \\
\text { hydrocephalus, CVS - cerebral vasospasm, DCl - delayed cerebral ischemia. }\end{array}$ & \\
\hline
\end{tabular}

Table 2: Multivariate logistic regression model for poor prognosis risk of poor-grade aSAH

\begin{tabular}{|lllll|}
\hline Variable included in Model & S.E & OR & $95 \% \mathrm{Cl}$ & $\mathrm{P}$ \\
\hline modified Fisher Grade (grade III, IV) & 0.515 & 2,972 & $1.083-8.156$ & 0.034 \\
\hline Age $(\geq 65)$ & 0.457 & 3.534 & $1.442-8.662$ & 0.006 \\
\hline Therapeutic Strategy (Conservation) & 0.694 & 5.078 & $1.303-19.790$ & 0.019 \\
\hline WFNS (Grade V) & 0.444 & 2.638 & $1.104-6.300$ & 0.029 \\
\hline DCI & 0.478 & 3.170 & $1.242-8.090$ & 0.016 \\
\hline SDH & 0.542 & 3.202 & $1.107-9.263$ & 0.032 \\
\hline Cerebral Herniation & 0.565 & 7.337 & $2.426-22.192$ & $<0.001$ \\
\hline Hosmer and Lemeshow test & & & & \\
\hline X & & & & 6.525 \\
\hline Degree of freedom & & & & 0.589 \\
\hline P & & & & \\
\hline
\end{tabular}

WFNS - World Federation of Neurosurgical Societies SDH - Shunt-dependent hydrocephalus, CVS cerebral vasospasm,

$\mathrm{DCl}$ - delayed cerebral ischemia 
Table 3: Aneurysmal Subarachnoid Hemorrhage Prognostic Scoring System (PASHPSS) derived from the $\beta$ coefficients

Variable included in Model

Categories

$\beta$ coefficient

Score

modified Fisher Grade

below grade II

0 (reference)

0

grade III, IV

1.09

1

Age

$\begin{array}{lll}\square 65 & 0 \text { (reference) } & 0 \\ \geq 65 & 1.26 & 1\end{array}$

Therapeutic Strategy

Coiling or Clipping

Conservation
0 (reference)

1.63
0

2

WFNS

Grade IV

0 (reference)

0

Grade V

0.97

1

DCI

Non - DCl
DCl

0 (reference)

0

1.15

1

SDH

Non - SDH

0 (reference)

0

SDH

1.16

1

Cerebral Herniation

\begin{tabular}{lll} 
Non - Cerebral Herniation & 0 (reference) & 0 \\
\hline Cerebral Herniation & 1.99 & 2
\end{tabular}

WFNS - World Federation of Neurosurgical Societies SDH - Shunt-dependent hydrocephalus, CVS cerebral vasospasm,

$\mathrm{DCl}$ - delayed cerebral ischemia 
Table 4: Risk of poor prognosis for low, intermediate, and high-risk individuals, according to the PASHPSS risk score

\begin{tabular}{|lllll|}
\hline $\begin{array}{l}\text { Risk } \\
\text { stratification }\end{array}$ & Score & $\begin{array}{l}\text { Observed risk } \\
\text { (validation cohort) }\end{array}$ & $\begin{array}{l}\text { Predicted } \\
\text { risk }\end{array}$ & OR (95\% Cl) \\
\hline $\begin{array}{l}\text { Low risk } \\
\text { stratification }\end{array}$ & $0-1$ & $19 \%$ & $11 \%$ & $\begin{array}{l}1 \\
\text { (Reference) }\end{array}$ \\
\hline $\begin{array}{l}\text { Moderate risk } \\
\text { stratification }\end{array}$ & $2-3$ & $48 \%$ & $51 \%$ & $\begin{array}{l}8.6(2.2- \\
\begin{array}{l}\text { High risk } \\
\text { stratification }\end{array}\end{array}$ \\
\hline
\end{tabular}

\section{Figures}

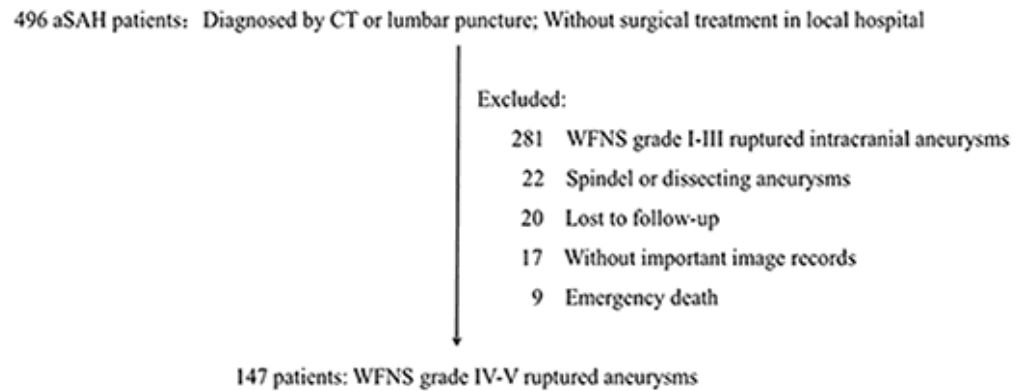

147 patients: WFNS grade IV.V ruptured ancurysms

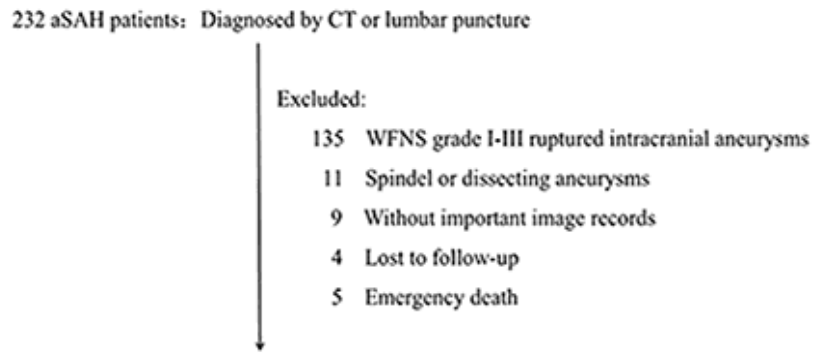

68 patients: WFNS grade IV.V ruptured ancurysms

\section{Figure 1}

Study flow diagram. aSAH, aneurysmal subarachnoid hemorrhage; CT, computerized tomography; WFNS, World Federation of Neurosurgical Societies. 

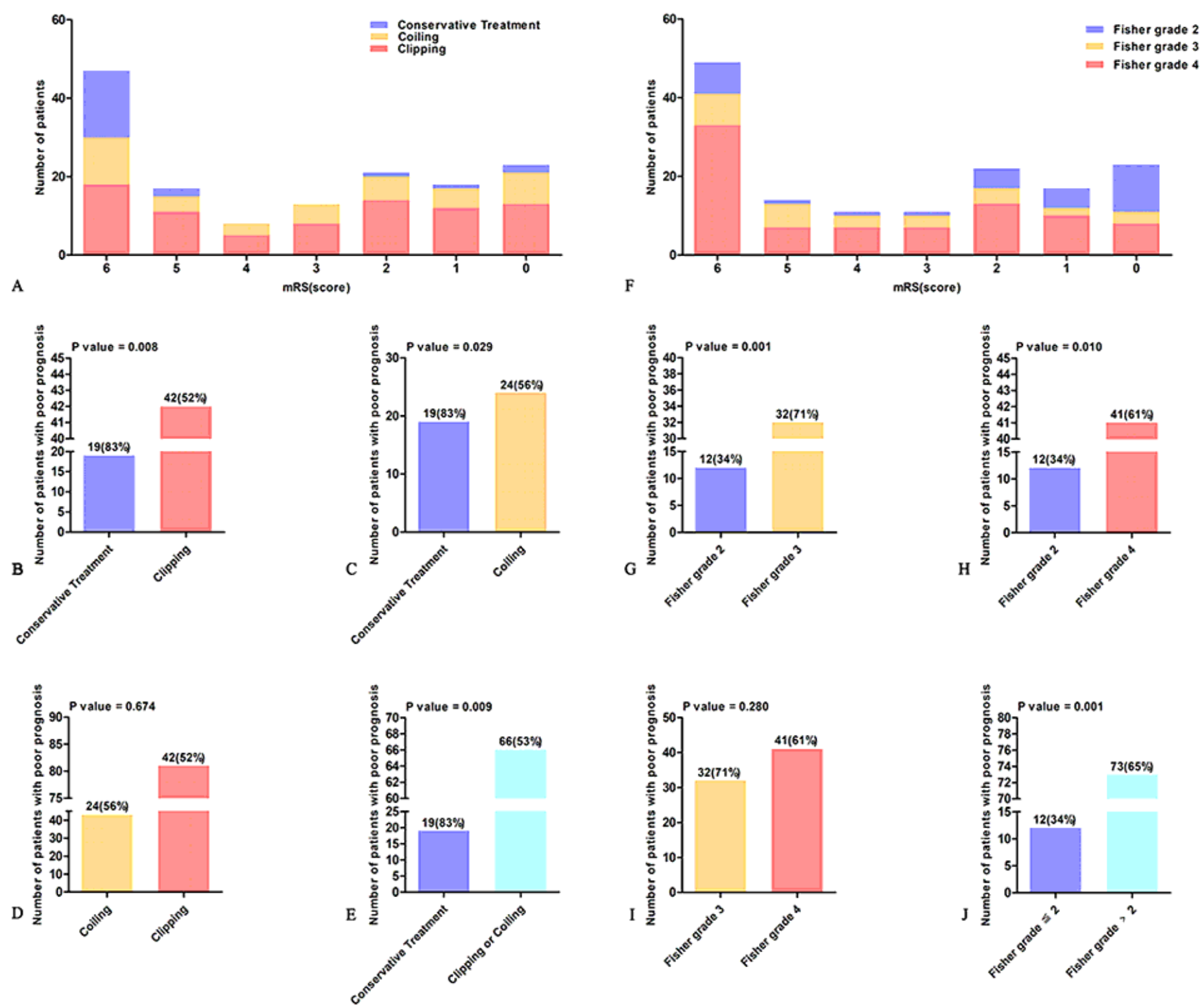

Figure 2

Figure 2 (A) shows the distribution of mRS score of 147 poor-grade aSAH patients accepted different treatment methods. The value above the histogram shows the number of patients with poor prognosis and the percentage of them, for instance, the interpretation of $19(83 \%)$ in Figure $2(B), 19(83 \%)$ patients with poor outcome among 33 patients who accepted conservation treatment. In Figure 2 (B, C, D, E) reflects the influence of different treatment methods on the prognosis of patients. Figure $2(\mathrm{~F})$ shows the distribution of mRS score of 147 poor-grade aSAH patients in different Fisher grade. Figure $2(G, H, I, J)$ reflects the influence of different Fisher grade groups on the prognosis of patients. 

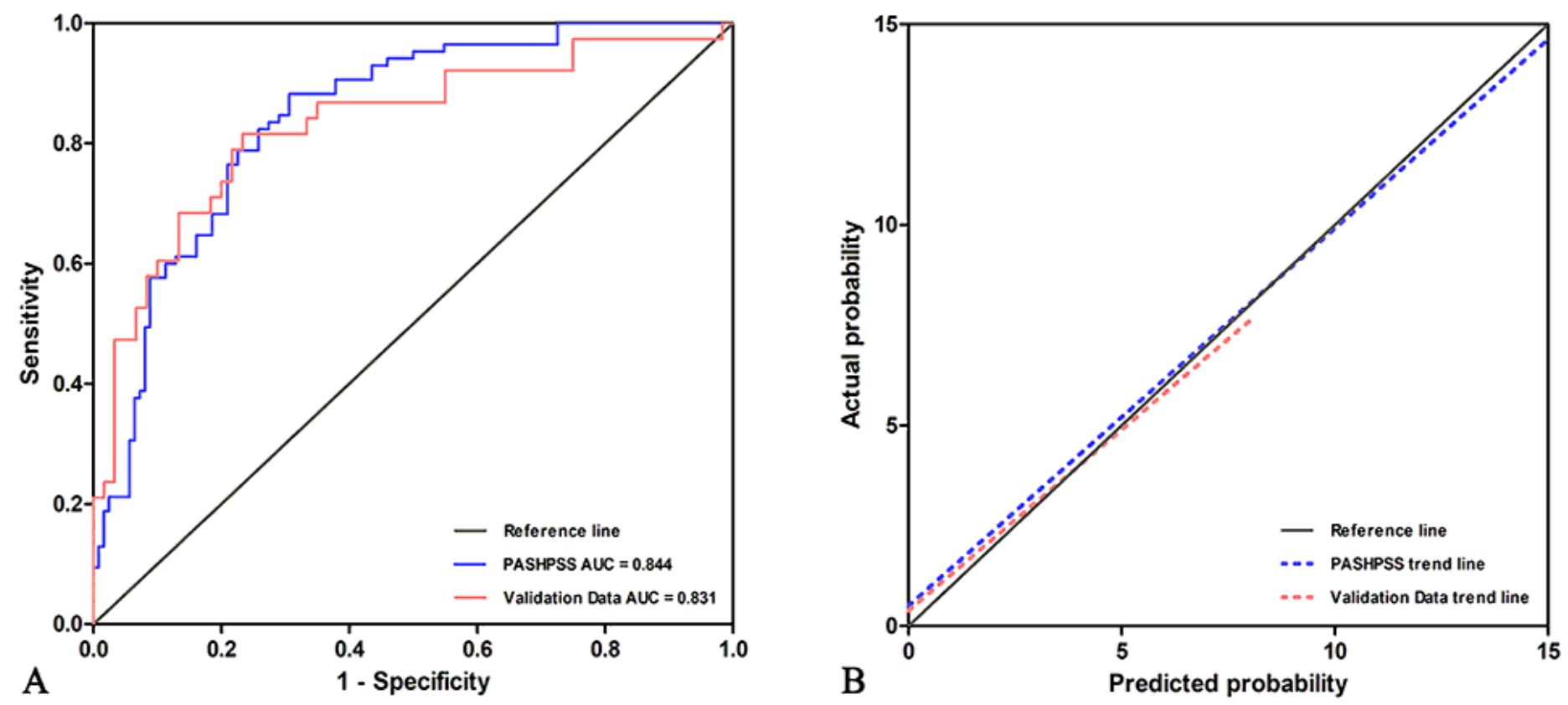

Figure 3

Figure $3(A)$ The AUC of the PASHPSS is $0.844(p<0.001 ; 95 \% \mathrm{Cl}, 0.778-0.909)$ in our center's modeling data, while it is $0.831(\mathrm{p}<0.001 ; 95 \% \mathrm{Cl}, 0.732$ - 0.929) in validation data. (B) A slope of 1 (45 degrees) with an intercept of 0 represents perfect calibration, the deviation from the reference line is smaller, the calibration is better. PASHPSS has a good calibration in derivation cohort and validation cohort.

\section{Supplementary Files}

This is a list of supplementary files associated with this preprint. Click to download.

- STROBEchecklist.pdf 